\title{
On the effect of the Froude number on the interface area of gravity-driven liquid rivulets
}

\author{
D. Sebastia-Saez ${ }^{\mathrm{a}}$, S. Gu ${ }^{\mathrm{a}}$, L. Könözsy ${ }^{\mathrm{b}}$, J.-U. Repke ${ }^{\mathrm{c}}$, H. Arellano-García ${ }^{\mathrm{a}, *}$ \\ ${ }^{a}$ Department of Chemical and Process Engineering, University of Surrey, Guildford, United \\ Kingdom, GU2 7XH \\ ${ }^{b}$ Centre for Computational Engineering Sciences, Cranfield University, Cranfield, United \\ Kingdom, MK43 OAL \\ ${ }^{c}$ Chair of Process Dynamics and Operations, TU Berlin, Berlin, Germany, D-10623
}

\begin{abstract}
The morphology of gravity-driven rivulets affects the mass transfer performance in gas separation processes, hence, the need for an improved knowledge on the hydrodynamics of this flow. It is well established that the interface area of the rivulets is determined by the balance between inertia and surface tension, i.e. the Weber number, which in light of the results presented here, are not the only parameters involved, but also the inclination of the plate has an effect on the balance of forces which determines the amount of gas-liquid interface area. The analysis of the interface area in rivulet flow demands, therefore, a more complete physical explanation for packing design purposes. In this work, we analyse the combined effect of both the inertia and the inclination of the plate in the interface area of liquid rivulets using CFD and the Volume-of-Fluid interface tracking method. As a result, we propose the use of the Froude number to provide a more complete physical explanation on the interface area formation of gravity-driven liquid rivulets.
\end{abstract}

Keywords: gravity-driven rivulet; interface area; gas separation; Froude number; CFD

\footnotetext{
* Corresponding author

Email address: h.arellano-garcia@surrey.ac.uk (H. Arellano-García)
} 


\section{Introduction}

Numerous investigations have been carried out toward simulating threedimensional multiphase flows inside packed gas absorbers, although the intricate geometry and the complex characteristics of the flow make the computational effort still too large. The modelling approach must, therefore, be divided into three length scales, which feed back each other (small-scale, meso-scale and large-scale) 1, 2. The whole column is considered at large-scale with the structured packing being modelled as a porous medium [3, 4]. The liquid distribution inside the absorber as well as the effect of geometric features on the flow are studied at this level. In meso-scale simulations, the computational domain is formed by a limited number of Representative Elementary Units (REU), which is the smallest repeated unit that forms the structured packing. The meso-scale approach represents the actual geometry of the packing and is used to assess the pressure drop per unit length [5, 6]. The link between meso- and largescale consists in that meso-scale simulations are used to determine the pressure drop coefficients, which are fed into the equation that defines the pressure drop through the porous medium, i.e. the Ergun equation. At small-scale, a 2D or $3 \mathrm{D}$ metallic plate is used to visualise the formation of the liquid film on the solid packing surface. That simple geometry allows to implement structured grids and interface tracking methods, such as the Volume-of-Fluid (VOF), at a reasonable computational cost [8, 9, 10]. The link between small- and mesoscale consists in the calculation of the velocity at the gas-liquid interface, which is used to obtain the wet pressure drop coefficients of the packing. On the other hand, interface tracking methods allow to visualise the liquid maldistribution phenomenon, which consists in the formation of droplets and rivulets within the column instead of the desired fully developed liquid film. Smallscale allows, therefore, to gain theoretical knowledge - otherwise difficult to get only with experimental methods - needed so as to improve the design of packed columns [13.

The relation between the forces acting on the fluid and the gas-liquid inter- 
face area has been investigated using small-scale CFD simulations [14. Three different liquid regimes depending on the liquid inlet velocity have been identified: trickling regime, rivulet regime, and full film flow regime. The trickling regime is characterised by the continuous detachment of droplets from the accumulation of liquid which builds up next to the liquid inlet. The rivulet flow regime is characterised by the formation of a zone next to the liquid inlet, where a developed liquid film forms, and two lateral and a central rivulet. In this physical process, the gas-liquid interface area increases with the liquid inlet velocity until the plate is fully covered. The Weber number allows to analyse the transition from one regime to another as the balance between the inertia of the liquid - which tends to spread the liquid over the surface - and the surface tension - which tends to minimise the gas-liquid interface. This balance has been used to explain the succession of braids and film areas as well [15].

The main hypothesis of this work is that, besides the initial inertia and the surface tension, also the effective component of the gravity along the plate, i.e. the inclination over the horizontal, has a considerable effect on the development of the gas-liquid interface area. Greater interface area are expected as the inclination of the plate over the horizontal plane increases due to the increment in the liquid velocity, however the visualisation of the velocity field within the liquid allows to determine that the momentum in the transversal direction of the flow is the factor causing the liquid spreading. The increase in the gravity component along the plate results in a decrement of the transversal momentum, and contrary to what was expected, a reduction in the interface area. Finally, the Froude number is proposed to quantify the combined effect of the liquid initial velocity and the plate inclination on the gas-liquid interface area. These conclusions can have an impact on the packing design, since smaller inclination is preferable from the point of view of the interface area. 


\section{Numerical Approach}

A 3D computational domain consisting of a smooth metallic surface - with dimensions $3 \mathrm{~cm} \times 0.5 \mathrm{~cm} \times 6 \mathrm{~cm}$ and total area of $18 \mathrm{~cm}^{2}$ - is used to study the rivulet flow. The schematic of the computational domain is shown in Figure 1 The observation plane in which the results of the figures 5, 6, 7 are taken is also depicted in the plot (at $5 \mathrm{~mm}$ from the lateral wall), as well as the inclination angle over the horizontal plane $\alpha$. It must be noted that the actual inclination of the solid wall in a packed column is the combination of the angle formed by the corrugation, and the angle that the ridges form with the horizontal plane. The values of $\alpha$ represented in this work do not correspond to any packing in particular, but have been selected as a representative value of the actual inclination of the packing wall over the horizontal plane. As for the boundary conditions, the liquid inlet is a $0.4 \mathrm{~mm}$ thick slot next to the wall. This choice is justified because the liquid film thickness in industrial applications varies from 0.2 to $0.4 \mathrm{~mm}$ (liquid loads from 10 to $50 \mathrm{~m}^{3} \cdot \mathrm{m}^{-2} \cdot \mathrm{h}^{-1}$ ) [1]. A pressure outlet is defined opposite to it. The rest of the boundaries have been defined as no-slip walls with a constant contact angle of $70^{\circ}$, which corresponds to the static contact angle of water on a steel plate. The relevant areas of the rivulet (braid, central rivulet, full developed liquid film, and front wave) are indicated to facilitate the analysis in Section 4

[Figure 1 around here]

The Volume of Fluid (VOF) model has been used for tracking the gas-liquid interface movement. The mass conservation can be written as

$$
\frac{\partial \rho}{\partial t}+\nabla \cdot(\rho \mathbf{u})=0,
$$

where the density $\rho$ is not constant throughout the computational domain, but varies according to

$$
\rho=\gamma_{l} \rho_{l}+\gamma_{g} \rho_{g},
$$

where $\gamma_{l}$ and $\gamma_{g}$ are the volume fraction of liquid and gas phases, respectively. 
The momentum equation is

$$
\frac{\partial(\rho \mathbf{u})}{\partial t}+\nabla \cdot(\rho \mathbf{u} \times \mathbf{u})=\rho \mathbf{g}-\nabla p+\nabla \cdot\left\{\mu\left[\nabla \mathbf{u}+(\nabla \mathbf{u})^{T}\right]\right\}+S_{\sigma},
$$

where $\mathbf{u}$ is the velocity field, $\mathbf{g}$ is the gravity field, $p$ is the hydrodynamic pressure, and the viscosity varies as

$$
\mu=\gamma_{l} \mu_{l}+\gamma_{g} \mu_{g}
$$

The surface tension momentum source term $S_{\sigma}$ is modelled according to the Continuum Surface Force (CSF) model introduced by Brackbill et al. (1992) as

$$
S_{\sigma}=\sigma \frac{\rho \kappa \nabla \gamma_{l}}{\frac{1}{2}\left(\rho_{l}+\rho_{g}\right)}
$$

where $\sigma$ is the surface tension, $\kappa$ represents the curvature of the interface between both phases, and the subscripts $l$ and $g$ denote liquid and gas respectively [16]. The curvature $\kappa$ is obtained as the divergence of the unitary vector perpendicular to the gas-liquid interface $\vec{n}$ according to

$$
\kappa=\nabla \cdot \vec{n}
$$

The contact angle $\theta$ is included to describe the liquid-solid adhesion. A correction for the normal direction is included as a function of $\theta$ as

$$
\vec{n}=\vec{n}_{\text {wall }} \cos \theta+\vec{t}_{\text {wall }} \sin \theta,
$$

where $\vec{n}_{\text {wall }}$ and $\vec{t}_{\text {wall }}$ are respectively the normal and tangent vectors to the wall, respectively.

The VOF model solves a single set of mass and momentum conservation equations (1)-(3) in the entirety of the computational domain.

Water and air have been set as the liquid and gas phase respectively, being the domain initially filled with air. A second-order upwind spatial discretisation scheme has been used along with the PRESTO pressure-correction algorithm. A 
variable time-step scheme has been chosen in order to keep the Courant number at a constant value $(C o=0.5)$, ensuring the stability of the transient simulations. The commercial software package ANSYS-FLUENT v14.0 has been used to run the calculations. ICEM-CFD has been used to generate the computational grids using the block strategy, with refinement near the wall boundaries. The characteristics of the grids are summarised in Table 2, The simulations have been performed in an HPC Cluster using $48 \mathrm{CPU}$ cores.

Six test cases with different values of the liquid inlet velocity and the inclination $\alpha$ over the horizontal plane have been performed. The six test cases are within the range of the rivulet flow regime. A factorial test design has been used to simulate the effect of both parameters on the interface area in a systematic manner. The liquid inlet velocity has three levels $(25,26$, and $30 \mathrm{~cm} / \mathrm{s})$ whereas the inclination over the horizontal plane has two levels $\left(45^{\circ}\right.$ and $\left.60^{\circ}\right)$. These test cases including the dimensionless Reynolds, Bond, Weber, and Froude numbers have been summarised in Table 5

[Table 1 around here]

The Bond number Bo quantifies the relative magnitude of the surface tension compared to gravity as

$$
B o=\frac{\Delta \rho g_{e f f} l^{2}}{\sigma},
$$

where $\Delta \rho$ denotes the density difference between both fluids, and $g_{e} f f$ is the effective acceleration of the gravitational force $\left(g_{e f f}=g \sin \alpha\right)$. The dimension of the plate in the $w$-direction $(6 \mathrm{~cm})$ has been used as the characteristic length $l$, i.e. see the coordinate axes disposition in Figure 1. Therefore, when the inclination angle $\alpha$ over the horizontal plane increases, the Bond number $B o$ increases as well due to the component of the gravity along the plate surface. On the other hand, the Weber number $W e$ quantifies the relative importance of the fluid inertia compared to surface tension as

$$
W e=\frac{\rho v^{2} l}{\sigma},
$$


where $v$ represents the liquid inlet velocity, and the dimension of the plate in the $w$-direction considered as the characteristic length $l$ as well. Therefore, when the liquid inlet velocity increases, the Weber number increases too.

The surface tension $\sigma$ between air and water $(0.072 \mathrm{mN} / \mathrm{m})$ remains unchanged in the present numerical study. Upon these conditions, if the density of the gas phase is neglected compared to the liquid phase, then the Froude number at liquid inlet conditions $\mathrm{Fr}$ gives the measure of the relative value between the inertia of the liquid and gravity. The Froude number $\mathrm{Fr}$ is assumed as being

$$
F r=\sqrt{\frac{W e}{B o}}=\frac{v}{\sqrt{g \sin \alpha l}} .
$$

The Froude number can, therefore, be used to study the combined effect of both the liquid inlet velocity and the gravity on the liquid distribution over the plate, when the surface tension is kept constant.

\section{Verification \& Validation (V\&V) Assessment}

Three grid-levels have been investigated with the specifications shown in Table 2. The grid refinement ratio $r=\sqrt[3]{N_{1} / N_{2}}=\sqrt[3]{N_{2} / N_{3}}$ is 1.2 in both refinement steps. The minimum grid spacing $h$ can also be found in Table 2 The grid spacing in the $v$-direction follows a logarithmic law so as to provide better refinement next to the solid wall.

[Table 2 around here]

The grid convergence study has been carried out relying on the generalised Richardson extrapolation proposed by Roache [19, 20]. The original work of Richardson [21] provided a method to estimate discretisation errors, and the

numerical solution of a variable $\phi$ is calculated - correcting an assumed exact solution $\phi_{\text {exact }}$ - by a series of terms as

$$
\phi=\phi_{\text {exact }}+g_{a} h+g_{b} h^{2}+\text { H.O.T. },
$$


where $h$ is the grid spacing, $g_{a}, g_{b}$ are functions that do not depend on $h$, and H.O.T. represents higher-order terms in the polynomial. The Richardson extrapolation requires the use of at least three grid-levels (coarse, medium, and fine) [22, 23, 24, however, it is also possible to use an appropriately chosen number of grid-levels [25, 26, 27]. The refinement ratio $r$ should be applied in each refinement step. The coarse, medium, and fine grids are denoted with the subscripts 3,2 , and 1 , respectively. The apparent order of convergence $p$ can be calculated by

$$
p=\frac{1}{\ln \left(r_{21}\right)}|\ln | \frac{\varepsilon_{32}}{\varepsilon_{21}}|+q(p)|,
$$

where absolute errors are computed as $\varepsilon_{32}=\phi_{3}-\phi_{2}$ and $\varepsilon_{21}=\phi_{2}-\phi_{1}$, and the function $q(p)$ is

$$
q(p)=\ln \left(\frac{r_{21}^{p}-s}{r_{32}^{p}-s}\right)
$$

where the parameter $s$ can be expressed as

$$
s=\operatorname{sgn}\left(\frac{\varepsilon_{32}}{\varepsilon_{21}}\right) .
$$

The extrapolated value at zero grid spacing $\phi_{\text {extr }}$ is then evaluated as

$$
\phi_{\text {extr }}=\frac{r_{21}^{p} \phi_{1}-\phi_{2}}{r_{21}^{p}-1}
$$

The Grid Convergence Index (GCI) is used to quantify the numerical accuracy. The $\mathrm{GCI}_{21}$ considering the fine and medium meshes is obtained as

$$
G C I_{21}=\frac{1.25 e_{21}}{r_{21}^{\tilde{p}}-1}
$$

where $\tilde{p}$ represents the average apparent order of convergence, and the relative error is

$$
e_{21}=\left|\frac{\phi_{1}-\phi_{2}}{\phi_{1}}\right| .
$$


The GCI and the relative error can also be obtained by considering the coarse and medium grids. It is important to note that the Richardson extrapolation can only be applied when the calculations lie within the asymptotic range. That condition is evaluated as

$$
\frac{G C I_{32}}{r^{p} G C I_{21}} \cong 1 .
$$

Table 3 summarises the results of the grid convergence study of the interface area using the three grids described in Table 5. The transient development of the interface area $f$ is shown in Figure 2, in which one can see a small difference between the results obtained with the three grids. The aim of this grid convergence study is to provide quantification of the grid discretisation errors, which in turn helps to choose the correct grid size for such time-consuming computations. Systematic grid convergence studies, with space discretisation error assessment in multiphase CFD simulations are scarce in the literature, and to the best of our knowledge, only Könözsy et al. (2009) and Sagadin et al. (2007) applied them to complex flows, such as spray dryers and solidification of metal ingots [28, 29]. Grid convergence assessments give also confidence on the CFD results, especially when there is a shortage of experimental data available for comparison. The fine grid is used to compute the results discussed in the following Section 4

[Table 3 around here]

[Figure 2 around here]

[Figure 3 around here]

The results contained in Table 3 show a progressive convergence of the results towards the theoretical extrapolated value at zero grid spacing. Figure 3 provides visualisation of the grid convergence. Most importantly, the asymptotic range condition has been checked, showing values approximately equal to 1, which means that the generalised Richardson extrapolation has been applied properly.

Comparison to experimental data found in the literature has been reported 
as well. Figure 4 shows the experimental results of Hoffmann et al. (2004, 2006) along with the six cases described in Table 5 [17, 18. A significant variation in the experimental results was reported, owing to imperfections on the solid surface and the hysteresis phenomenon studied by Iso and Chen (2011), who observed that the interface area for a given value of the liquid flow rate depends on the previous history [32]. The Reynolds number is used as a measure of the inlet velocity, although all of the data were obtained with water as the liquid phase. A direct relation between the Reynolds number and the interface area is observed. The plot depicts the transition between the three regimes (vertical red lines). The transition between the trickling and the rivulet flow is approximated according to the values stated by Xu et al. (2012) and Singh et al. (2017), who established the threshold at $R e \approx 60$ [33, 34]. The results reported in this work are limited to the range of the rivulet flow regime, since it is in this range that the system is more sensitive to small variations in the interface area (the boundaries between regimes are also represented in Figure 4). The full film flow regime has been discarded in this work, since it does not appear in structured packed columns, whereas results lying within the trickling flow boundaries make the comparison between cases more difficult as the continuous detachment of droplets imply significant variations in the transient behaviour of the interface area. The inlet plot is a zoom which helps to visualise the data obtained with the CFD simulations. The results of this work lie between the values obtained by Hoffmann et al. (2004, 2006), although the lack of experimental error bars makes the validation of our results difficult, hence the comprehensive grid convergence study.

[Figure 4 around here]

\section{Results and discussion}

The effect of the inertia and the inclination of the plate on the interface area has been reported in Figure 4, in which the results have also been compared to experimental data. Our results are grouped in two different series according 
to their Bond number. The effect of increasing the liquid inlet velocity can be assessed considering each series of data separately. Both show that an increase of the liquid inlet velocity, represented in the plot by the Reynolds number (the liquid and the gas are water and stagnant air respectively in all of the cases), results in better spreading. The effect of the inclination of the plate can also be seen in the plot. The series with the greater $B o$ number $(B o=423.4)$, i.e. greater inclination of the plate, lies below the series with $B_{o}=344.9$, meaning that greater inclination gives rise to less liquid spreading. At equal flow rates, a greater inclination over the horizontal gives rise to greater accelerations and therefore, greater velocities along the plate, which should decrease the characteristic length of the rivulets, in order to keep the flow rate constant, resulting in less area of the plate covered by the liquid. The visual representation of the extension achieved by the liquid film depicted in Figure 5 helps with visualising the final extension of the area in which full film flow is attained. The shape of the front wave can also be remarked in the plot, marking the end of the full film flow area and being characterised by a stagnation of the velocity in the longitudinal direction and a change in the direction of the transversal velocity. The distance from the wall and from the liquid inlet are normalised by the correspondent dimension of the plate $(5 \mathrm{~mm}$ and $6 \mathrm{~cm}$, respectively). The plot is obtained in the longitudinal plane at $5 \mathrm{~mm}$ from the lateral wall, and thus, avoiding both the lateral and the central rivulet in order to focus strictly on the full film area. The length of the full film area is included in the plot showing significant changes between the cases. If one compares, for instance, cases $\mathrm{A}$ and $\mathrm{B}$, which have the same liquid inlet velocity $(30 \mathrm{~cm} / \mathrm{s})$ and only differ in the inclination of the plate $\left(45^{\circ}\right.$ in case $\mathrm{A}$ and $60^{\circ}$ in case $\mathrm{B}$, that is, $33 \%$ increase of angle), one can appreciate a $10.43 \%$ decrease of the reach of the full film flow area, which translates in a $10.92 \%$ loss of interface area. Moreover, if one focuses attention in a pair of cases with the same inclination and different velocities (for instance, cases $\mathrm{A}$ and $\mathrm{D}$, both with $45^{\circ}$ inclination, and $30 \mathrm{~cm} / \mathrm{s}$ for case $\mathrm{A}$ and $25 \mathrm{~cm} / \mathrm{s}$ case $\mathrm{D}$, that is a $16.7 \%$ decrease), then there is a $38.5 \%$ decrease in the length of the film flow area and a $17 \%$ decrease 
in the interface area. The effect of the change in liquid inlet velocity is more pronounced than that of the change in the plate inclination, although the latter is still notable. This means that significant improvements in the mass transfer performance could be attained by simply reducing the inclination angle of the plate, provided that the total mass transfer rate is the result of multiplying the mass flux through the gas-liquid interface times the interface area.

The effect of the plate inclination, i.e. the effective gravity component along the plate, is therefore opposite to what is expected given the relation between the inertia of the fluid and the liquid spreading. The reason of this behaviour can be further clarified by looking into the velocity components in the fully developed film area. To this end, the work of Mertens et al. (2004), who explained the formation of successive braids in gravity-driven rivulets is of help [15]. The authors discussed that the inertia of the fluid spreads the liquid towards both sides of the centre line of the domain beyond the equilibrium with the surface tension leading to the formation of regions with fully developed liquid films. After that, the surface tension brings the liquid back towards the centre line of the domain. The two streams flowing in opposite directions bounce eventually at the centre line, giving way to the formation of a liquid accumulation called braid. The process repeats itself until damped by dissipative forces, after which a rounded-shaped rivulet appears. This phenomenon can also be observed in Figure 1. where the main parts of the flow have been depicted. Initially, a region with a fully developed film forms, giving gradually way to the formation of a front wave in which the flow must be directed towards the centre line in order to give rise to the central braid and the subsequent rivulet. Consequently, this continuous readjustment caused by the transversal movement of the liquid suggests that there must be changes in the direction of the transversal velocity component within the liquid phase. The transversal component of the velocity should therefore be directed towards the centre line of the domain in those areas in which the liquid withdraws towards the centre, and away from the centre line when there is liquid spreading and full film areas, with the turning point placed in the vicinity of the front wave. The transversal $\left(v_{u}\right)$ and longitudinal $\left(v_{w}\right)$ 
velocity components within the fully developed film are represented in Figure 6 and Figure 7 . The subplots in the same row contain data with the same value of the Weber number and different value of the Bond number so as to assess the effect of the plate inclination on both velocity components. The values shown correspond to quasi-steady conditions (last data point in Figure 2). The velocities (normalised by the liquid inlet velocity) are taken along the line parallel to the surface of the plate (distance from the plate constant at $y=0.2 \mathrm{~mm}$ ) and contained in the plane depicted in Figure 1. The plots also show which values correspond to the gas and the liquid phase, respectively. Non-zero values are observed in the gas phase owing to momentum exchange between both phases. Also, gas recirculation through the pressure outlet results in a notable negative value observed near the outlet in Figure 7 . The three subplots on the left-hand side encompass the entire length of the domain so as to remark the spike in the profile which appears next to the front wave. The three plots on the right-hand side show only the values in the vicinity of the front wave with the objective of conveying the change in the direction of the velocity clearly. The boundary between the liquid phase and the gas phase is marked by the vertical line of the same colour than the respective data series. In all of the instances, the transversal velocity (Figure 6 presents negative values in the majority of the range within the liquid, i.e. negative values mean that the flow is directed towards the periphery of the domain, which would support the qualitative description of Mertens et al. (2004), according to which the liquid spreads sidewards in that area. In the front wave, however, the trend reverses towards positive values of the transversal velocity $v_{u}$, i.e. directed to the centre line of the domain, according to the reference frame depicted in Figure 1, with the formation of a spike in the graph. That peak in the transversal velocity $\left(v_{u}\right)$ results in the liquid phase feeding the formation of the central braid and needs necessarily to give rise to a decrease in the longitudinal component of the velocity so as keep the momentum constant, as observed in Figure 7. The longitudinal component of the velocity drops to zero in the area of the front wave, meaning that the momentum of the liquid phase is entirely 
directed toward the centre of the domain, hence the formation of a pronounced spike in the transversal component. There is therefore, a stagnation of the liquid phase in the longitudinal direction owing to the movement being damped by the cohesion of the liquid. This proves the existence of a readjustment between the inertia and the surface tension around an equilibrium point that determines the extension of the liquid film and therefore, the gas-liquid interface area.

Therefore, greater values of the longitudinal component are expected for greater inclination, provided that the effective component of gravity increases in such case. One can confirm this hypothesis by looking at the zoom of the velocity profiles on the right-hand side of Figure 7 . In all of the instances, the greatest longitudinal components of the velocity correspond to the inclination $\alpha=60^{\circ}$ instead of $\alpha=45^{\circ}$. Conversely, the plots on the right-hand side of Figure 6 show greater absolute values of the transversal component $v_{w}$ at $B o=344.9$, and $\alpha=$ $45^{\circ}$. This is explained by the conservation of momentum, which results thus in smaller values of the transversal component $v_{w}$ to compensate the increase in the longitudinal component $v_{u}$ caused by the additional momentum source termdue to gravity - in the longitudinal projection of the momentum conservation equation. Greater inclination of the plate over the horizontal, therefore, directs the flow towards the central rivulet faster. At equal initial velocity, if the main interest is to achieve good spreading, then one needs not to force the linear momentum of the liquid in only one direction, but to let both components develop without the effect of gravity. If the later is eliminated, then the final reach of the liquid phase comes determined by the liquid flow rate entering the domain, that is, the liquid inlet velocity, as proved by the well-known relation between the Reynolds number and the interface area.

The effect of the combined effect of the initial inertia and the inclination of the plate on the liquid spreading has been studied in this work, although both of them can be reduced to the same ultimate cause, that is, directing the flow sideways instead of along the flow direction. This might be achieved by either increasing the liquid flow rate or decreasing the inclination of the plate. This suggests the existence of a direct relationship between the Froude 
number and the gas-liquid interface area, which would provide a more complete description of the phenomena taking place than the Reynolds or the Weber number. Figure 8 depicts the direct relation between both parameters in the range studied, including the error bars that account for the grid discretisation errors. The graph shows a steady increase of the interface area with the Froude number, although a greater step can be observed between cases A and B, and the rest owing to the difference between the liquid inlet velocity of those two cases and the rest. This direct relation found might be used for design purposes to predict the interface area as a function of the inclination of the plate and the initial inertia of the liquid.

\section{Conclusions}

A Computational Fluid Dynamics (CFD) approach has been used to study the hydrodynamics of the liquid distribution over inclined solid surfaces, with application in engineering problems such as gas absorption in structured packings. The relation between the liquid flow rate and the interface area has been reported in the literature, and explained relying on the equilibrium between the inertia of the liquid and the surface tension, which tends to minimise the gas-liquid interface area. The successive formation of braids and full film areas is also explained on this basis. In this work, evidence to that behaviour has been provided by studying the velocity components in the successive film-braid areas obtained by CFD. The data reported show that the transversal velocity is directed towards the periphery of the domain along the full film area, giving way to the extension of the liquid phase over the solid surface. Conversely, there is an upturn in the front wave that results in the velocity being directed towards the centre line. That leads eventually to the formation of a central braid as a result of both streams colliding in the centre line.

The results also show that, at equal liquid inlet velocity, smaller inclination of the plate over the horizontal gives rise to enhanced gas-liquid interface area, which is beneficial to mass transfer. A deeper analysis of the velocity profiles 
elucidates the reason of this behaviour. The inclusion of the additional momentum source term in the momentum conservation equation along the longitudinal direction, i.e. parallel to the flow, results in the velocity components in this direction being greater as the effective gravity component along the plate surface increases. As per conservation of momentum, there should be a decrease in the transversal component when the liquid accelerates in the longitudinal direction. In the light of these results, one can conclude that the transversal component of the velocity is the ultimate cause of liquid spreading, and therefore, it is interesting from the design point of view to ensure that the transversal component of the velocity is high enough so as to enhance liquid spreading.

The Froude number at the liquid inlet has been used to quantify the combined effect of the initial inertia and the inclination of the plate has proved to have a direct effect on the interface area as well. At same inclination value, greater liquid inlet velocity gives rise to greater liquid spreading. Similarly, at equal liquid inlet velocity, it is preferable to have small inclination over the horizontal in order to get more gas-liquid interface area. It is therefore interesting regarding the design, to ensure small plate inclination and large inlet velocity so as to increase the gas-liquid interface area in rivulet flow. This work provides

then directions as to how to optimise the design of rivulet flows based on the interface area.

\section{Acknowledgements}

The authors gratefully acknowledge the financial support from the EPSRC: Project grant EP/J020184/1; and FP7 Marie Curie iComFluid project grant 312261.

\section{References}

[1] L. Raynal, A. Royon-Lebeaud, A multi-scale approach for CFD calculations of gas-liquid flow within large size column equipped with structured packing, Chem. Eng. Sci. 62 (2007) 7196-7204. 
[2] B. Sun, L. He, B. Liu, F. Gu, C. Liu, A new multi-scale model based on CFD and macroscopic calculation for corrugated structured packing column, AlChE J. 59 (2013) 3119-3130.

[3] J. Kim, D. Pham, Y. Lim, Gas-liquid multiphase computational fluid dynamics (CFD) of amine absorption column with structured-packing for $\mathrm{CO}_{2}$ capture, Comput. Chem. Eng. 88 (2016) 39-49.

[4] M. Fourati, V. Roig, L. Raynal, Liquid dispersion in packed column: Experiments and numerical modelling, Chem. Eng. Sci. 100 (2013) 266-278.

[5] L. Armstrong, S. Gu, K. Luo, Dry pressure drop prediction within Montzpak B1-250.45 packing with varied inclination angles and geometries, Ind. Eng. Chem. Res. 52 (2013) 4372-4378.

[6] W. Said, M. Nemer, D. Clodic, Modeling of dry pressure drop for fully developed gas flow in structured packing using CFD simulations, Chem. Eng. Sci. 66 (2011) 2107-2117.

[7] Y. Haroun, L. Raynal, P. Alix, Prediction of effective area and liquid holdup in structured packings by CFD, Chem. Eng. Res. Des. 92 (2014) 22472254 .

[8] D. Sebastia-Saez, S. Gu, P. Ranganathan, K. Papadikis, 3D modeling of hydrodynamics and physical mass transfer characteristics of liquid film flows in structured packing elements, Int. J. Greenhouse Gas Control 19 (2013) 492-502.

[9] D. Sebastia-Saez, S. Gu, P. Ranganathan, K. Papadikis, Micro-scale CFD modeling of reactive mass transfer in falling liquid films within structured packing materials, Int. J. Greenhouse Gas Control 33 (2015) 40-50.

[10] P. Valluri, O. Matar, G. Hewitt, M. Mendes, Thin film flow over structured packings at moderate Reynolds numbers, Chem. Eng. Sci. 60 (2005) 19651975. 
[11] Y. Haroun, D. Legendre, L. Raynal, Direct numerical simulation of reactive absorption in gas-liquid flow on structured packing using interface capture method, Chem. Eng. Sci. 65 (2010) 351-356.

[12] M. Fourati, V. Roig, L. Raynal, Experimental study of liquid spreading in structured packings, Chem. Eng. Sci. 80 (2012) 1-15.

[13] M. Kohrt, I. Ausner, G. Wozny, J. Repke, Texture influence on liquidside mass transfer, Chemical Engineering Research and Design 89 (2011) $1405-1413$.

[14] Y. Iso, J. Huang, M. Kato, S. Matsuno, K. Takano, Numerical and experimental study on liquid film flows on packing elements in absorbers for post-combustion $\mathrm{CO}_{2}$ capture, Energy Procedia 37 (2013) 860-868.

[15] K. Mertens, V. Putkaradze, P. Vorobieff, Braiding patterns on an inclined plane, Nature 430 (2004) 165.

[16] J. Brackbill, D. Kothe, C. Zemach, A continuum method for modelling surface tension, J. Comput. Phys. 100 (1992) 335-354.

[17] A. Hoffmann, I. Ausner, J. Repke, G. Wozny, Fluid dynamics in multiphase distillation processes in packed towers, Comput. Aided Chem. Eng. 18 (2004) 199-204.

[18] A. Hoffmann, I. Ausner, J. Repke, G. Wozny, Detailed investigation of multiphase (gas-liquid and gas-liquid-liquid) flow behavior on inclined plates, Chem. Eng. Res. Des. 84 (2006) 147-154.

[19] P. Roache, Perspective: A method for uniform reporting of grid refinement studies, ASME J. Fluids Eng. 116 (1994) 405-413.

[20] P. Roache, Verification and validation in computational science and engineering, Hermosa, Albuquerque, NM, 1998.

[21] L. Richardson, The approximate arithmetical solution by finite differences of physical problems involving differential equations, with an application 
to stresses in a masonry dam, Philos. Trans. R. Soc. London, Ser. A 210 (1911) 307-357.

[22] C. Freitas, The issue of numerical uncertainty, Appl. Math. Modell. 26 (2002) 237-248.

[23] K. Elsayed, C. Lacor, CFD modeling and multi-objective optimization of cyclone geometry using desirability function, artificial neural networks and genetic algorithms, Appl. Math. Modell. 37 (2013) 5680-5704.

[24] A. Khelil, H. Naji, L. Loukarfi, M. H. Meliani, M. Braikia, Numerical simulation of the interactions among multiple turbulent swirling jets mounted in unbalanced positions, Appl. Math. Modell. 40 (2016) 3749-3763.

[25] C. Marchi, L. Araki, A. Alves, R. Suero, S. Gonçalves, M. Pinto, Repeated Richardson extrapolation applied to the two-dimensional Laplace equation using triangular and square grids, Appl. Math. Modell. 37 (2013) 46614675 .

[26] C. Marchi, L. Novak, C. Santiago, A. da Silveira-Vargas, Highly accurate numerical solutions with repeated Richardson extrapolation for 2D Laplace equation, Appl. Math. Modell. 37 (2013) 7386-7397.

[27] C. Marchi, M. Martins, L. Novak, L. Araki, M. V. Pinto, S. de Fátima Tomazzoni Gonçalves, D. Moro, I. da Silva Freitas, Polynomial interpolation with repeated Richardson extrapolation to reduce discretization error in CFD, Appl. Math. Modell.

[28] L. Könözsy, A. Ishmurzin, F. Mayer, M. Grasser, M. Wu, A. Ludwig, Numerical investigation of grid influence on formation of microsegregation, Int. J. Cast Met. Res. 22 (2009) 175-178.

[29] G. Sagadin, M. Hriberšek, A multistage spray drying model for zeolite 4A - water suspensions in a counter-current spray dryer, Int. J. Heat Mass Transfer 108 (2017) 1220-1228. 
[30] J. Hu, X. Yang, J. Yu, G. Dai, Numerical simulation of carbon dioxide $\left(\mathrm{CO}_{2}\right)$ absorption and interfacial mass transfer across vertically wavy falling film, Chem. Eng. Sci. 116 (2014) 243-253.

[31] J. Cooke, L. Armstrong, K. Luo, S. Gu, Adaptive mesh refinement of gasliquid flow on an inclined plane, Comput. Chem. Eng. 60 (2013) 297-306.

[32] Y. Iso, X. Chen, Flow transition behavior of the wetting flow between the film flow and rivulet flow on an inclined wall, Journal of Fluids Engineering 133 (2011) 091101.

[33] R. Singh, J. Galvin, G. Whyatt, X. Sun, Breakup of a liquid rivulet flowing over an inclined plate: Identification of a critical weber number, Physics of Fluids 29 (2017) 052101.

[34] Y. Xu, J. R. J. Yuan, G. Wozny, CFD study on liquid flow behavior on inclined flat plate focusing on effect of flow rate, Engineering Applications of Computational Fluid Mechanics 6 (2012) 186-194. 


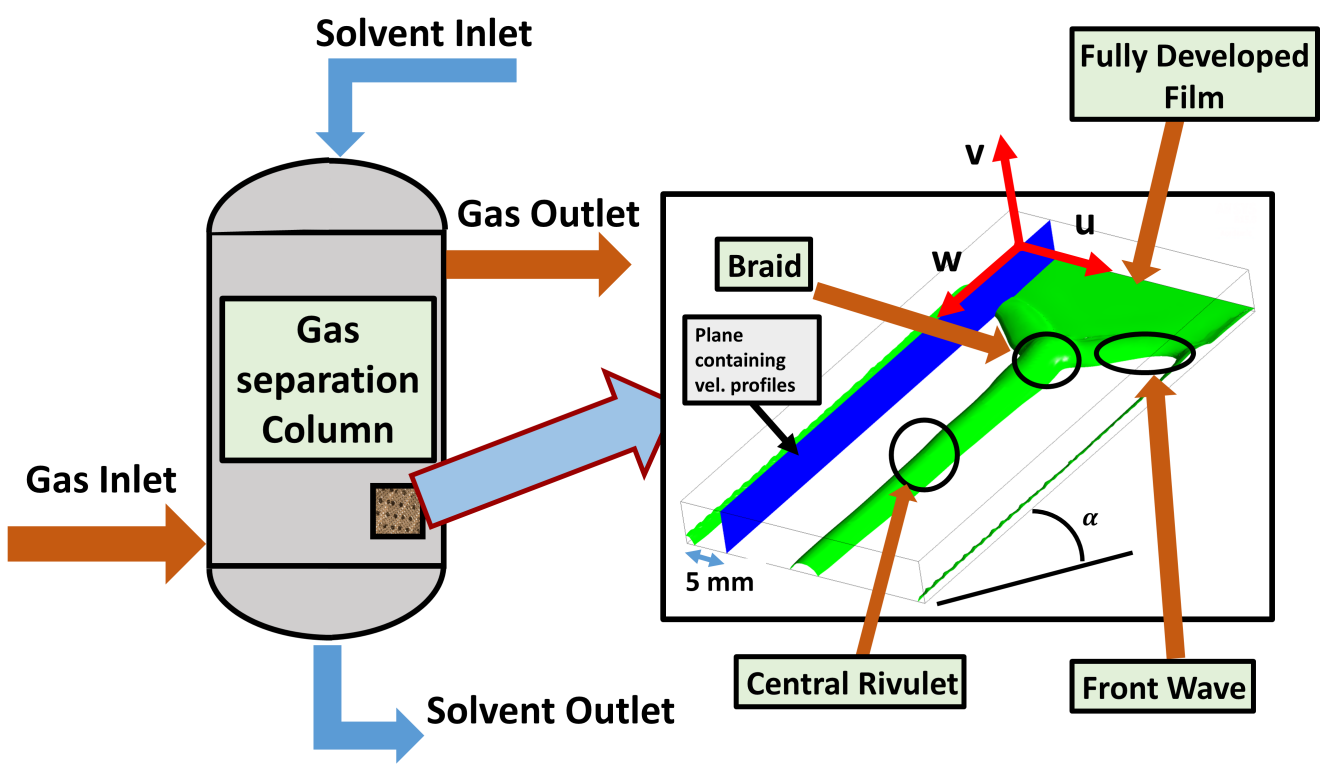

Figure 1: Schematic illustration of the relation between a structured packing column and the computational domain at small-scale. The sketch on the right-hand side shows the simplified computational domain (an inclined plate with dimensions $3 \mathrm{~cm} \times 0.5 \mathrm{~cm} \times 6 \mathrm{~cm}$ and a total area of $18 \mathrm{~cm}^{2}$ ). The relevant areas of the liquid pattern formed-defined as the isosurface with volume fraction $\gamma=0.5$ - are indicated; namely central rivulet, front wave, braid, and fully developed film. The plot also depicts the observation plane in which the results of Figures 5 6] and 7 are taken. 

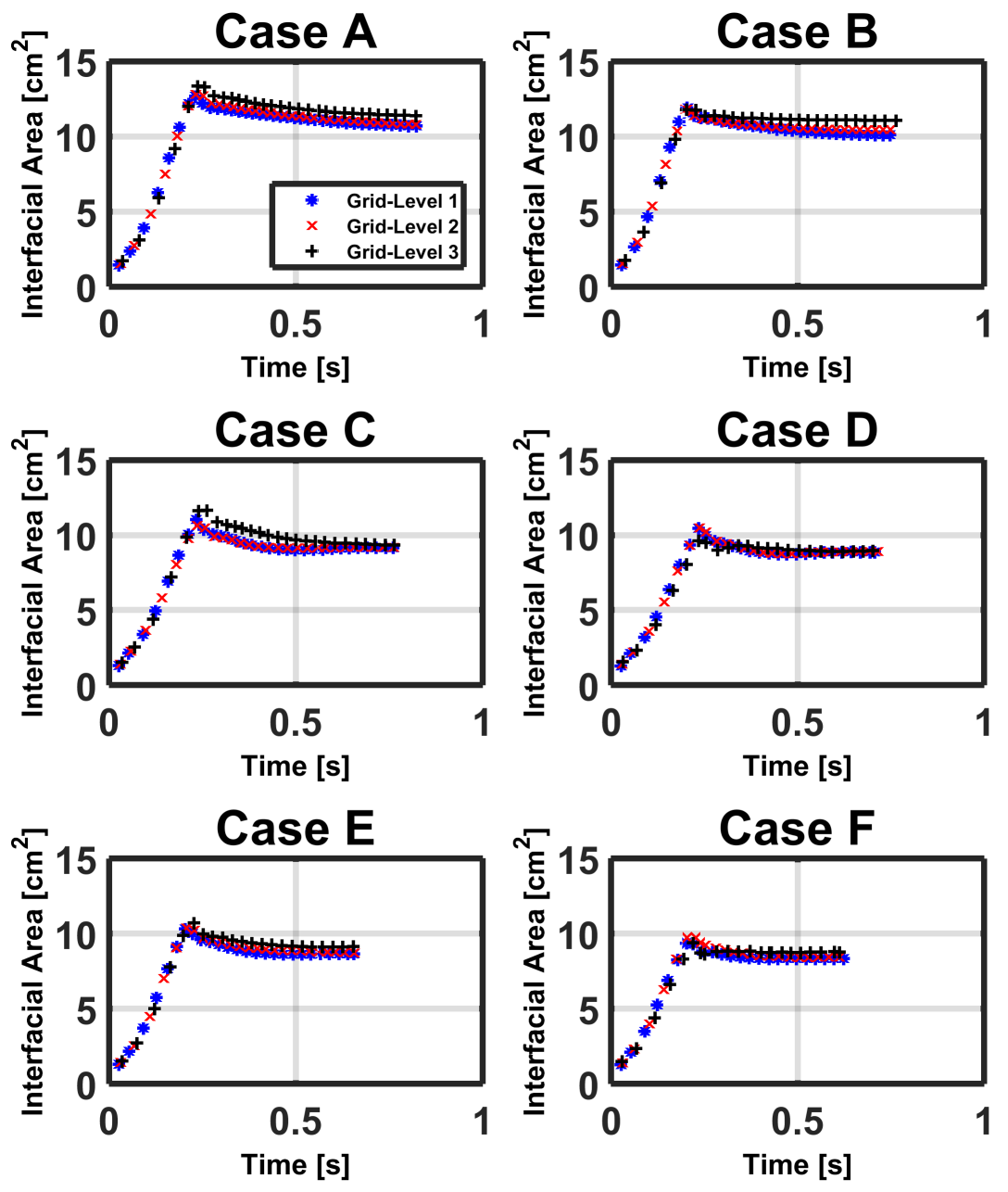

Figure 2: Transient evolution of the interfacial area $f$ for the six cases tested, using the three grid levels. The legend is only represented in one of the subplots for simplicity. 


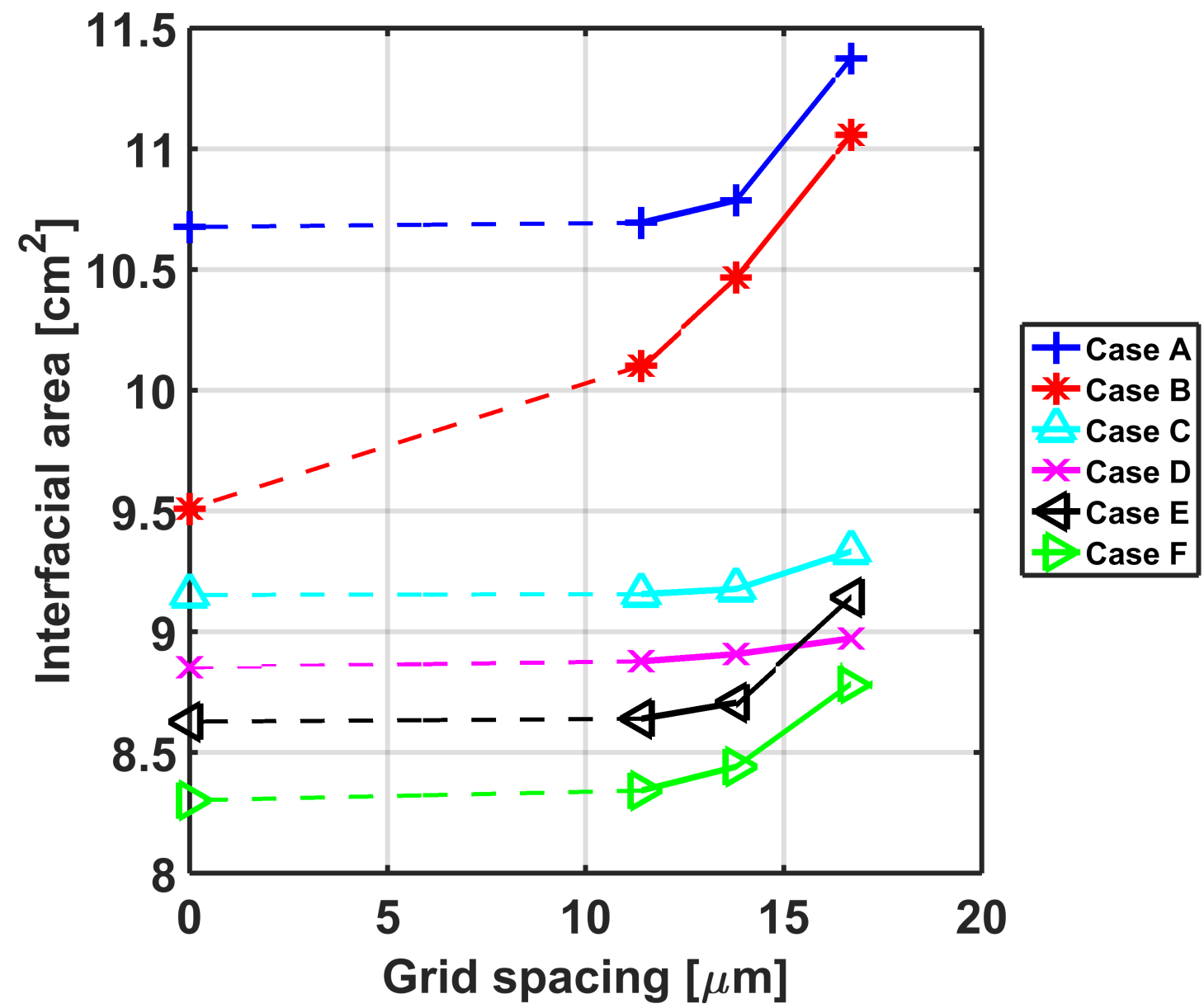

Figure 3: Grid convergence plot showing the effect of the grid spacing on the gas-liquid interface area $f$ for the six cases tested (cases described in Table 5). The continuous line links the data obtained with the CFD simulations. The dashed line represents the extension towards the extrapolated value obtained with the Richardson extrapolation. 


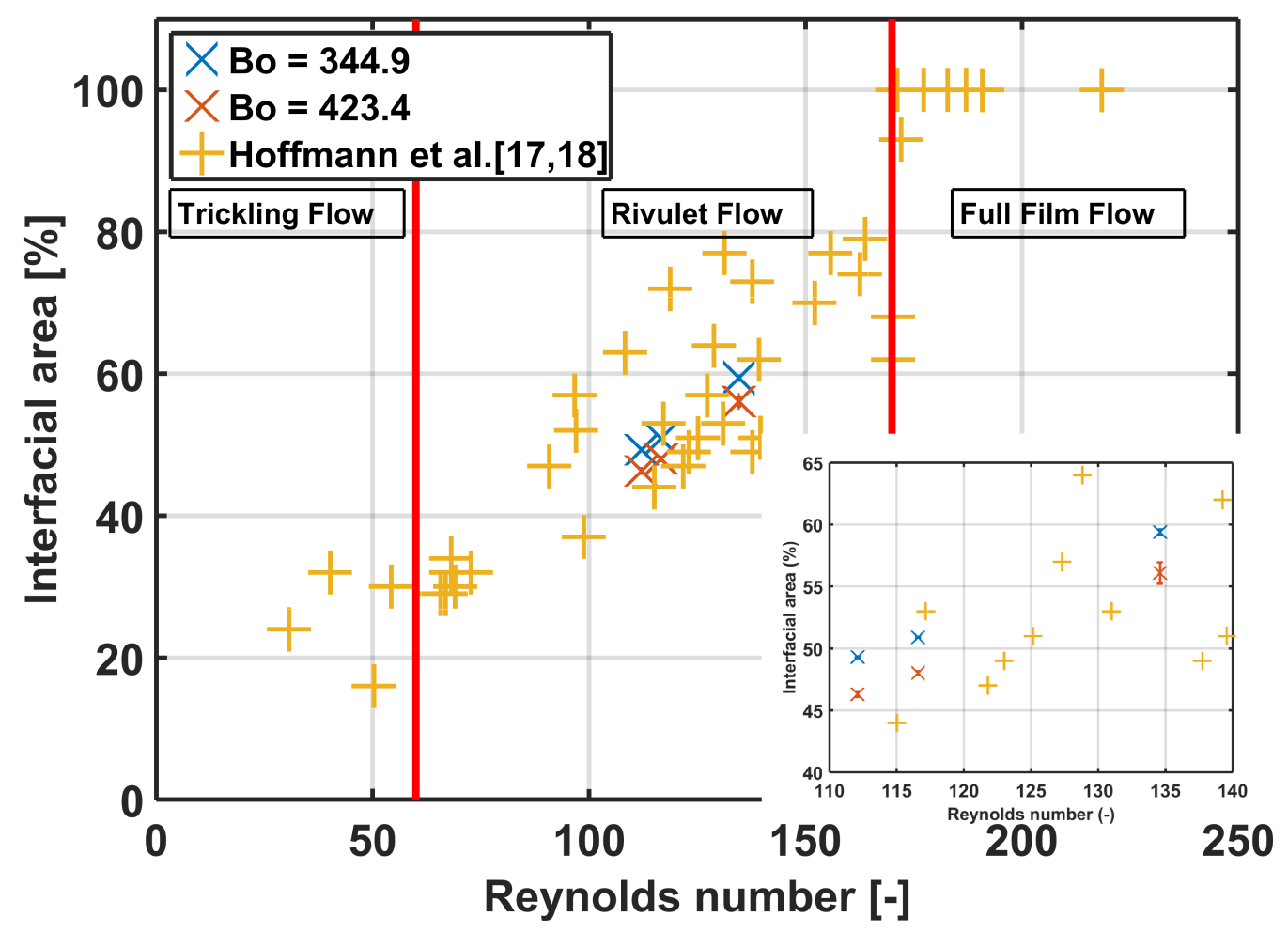

Figure 4: Comparison of the present CFD simulations with the experimental results of Hoffmann et al. 17 18, highlighting the three regimes reported in the literature: trickling flow, rivulet flow, and full film flow. The inset figure represents a zoom for better visualisation of the results of this work. The Reynolds number is used as a measure of the inertia of the liquid, being defined as $R e=\frac{\rho v l}{\mu}$, where the characteristic length $l$ is the thickness of the thin slot that constitutes the liquid inlet $(0.4 \mathrm{~mm})$, and $\mu$ is the dynamic viscosity. 

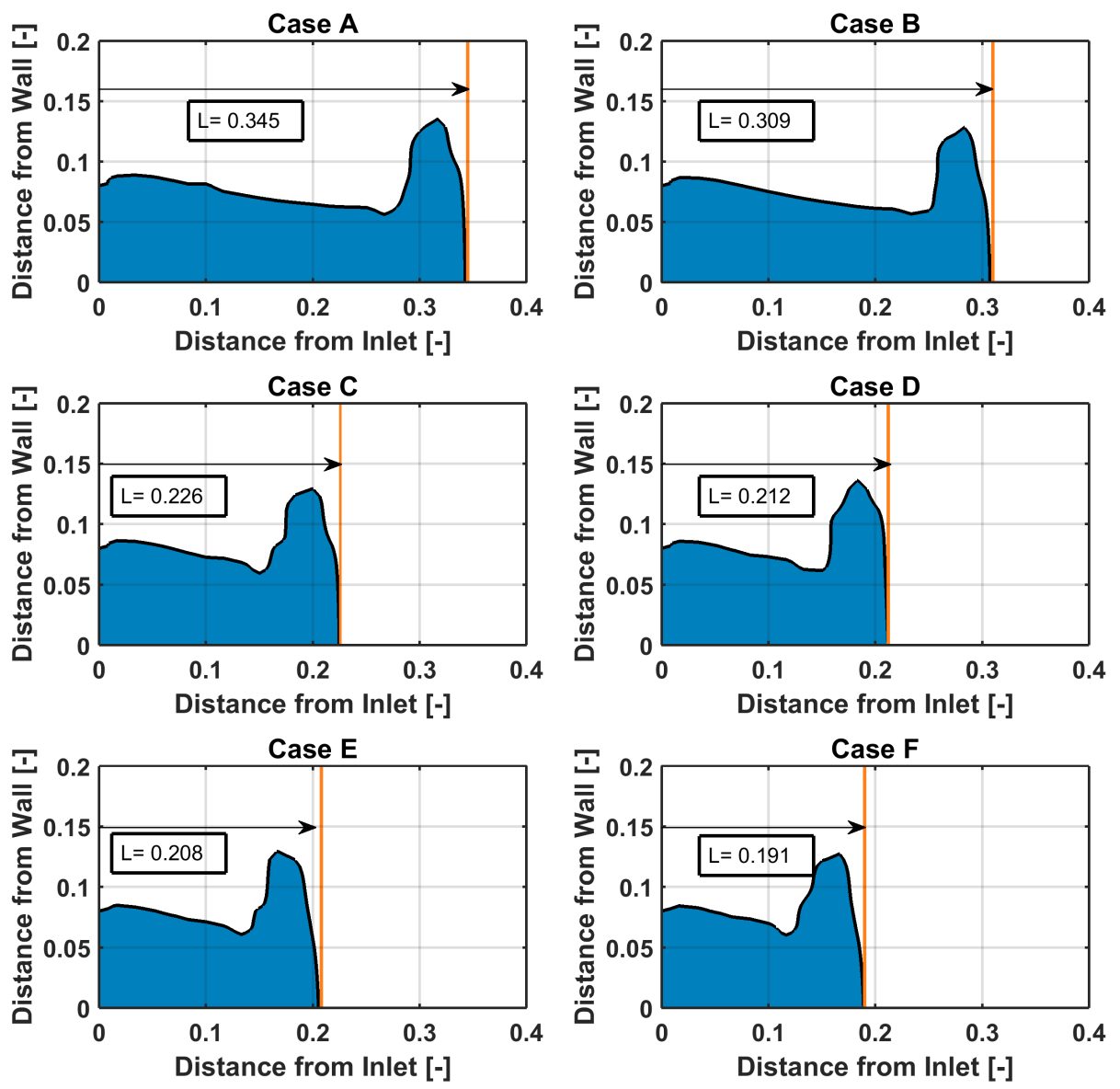

Figure 5: Liquid phase contours of the liquid phase within the observation plane shown in Figure 1 at volume fraction $\gamma=0.5$ and a distance of $5 \mathrm{~mm}$ from the lateral boundary. The contours help to visualise the extension of the liquid film and the shape of the front wave (blue denotes liquid phase). 

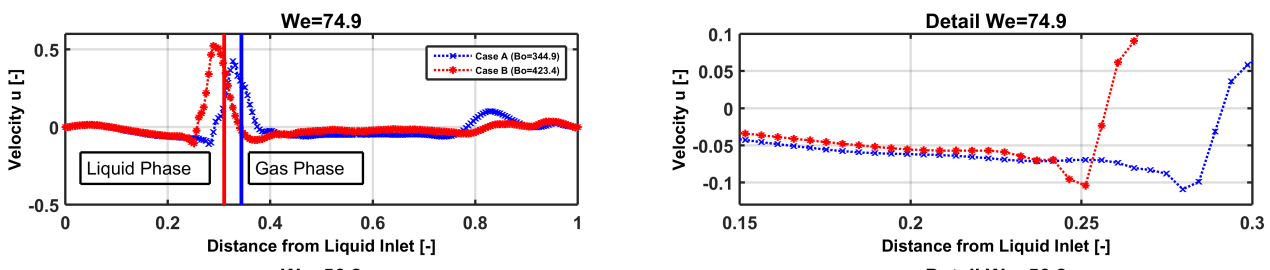

$\mathrm{We}=56.2$

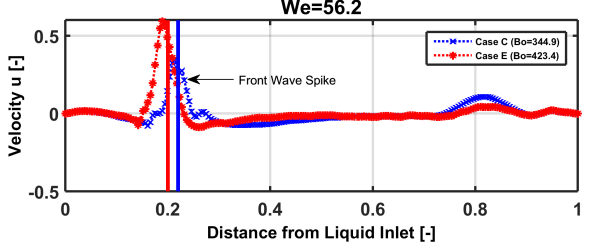

Detail We $=56.2$

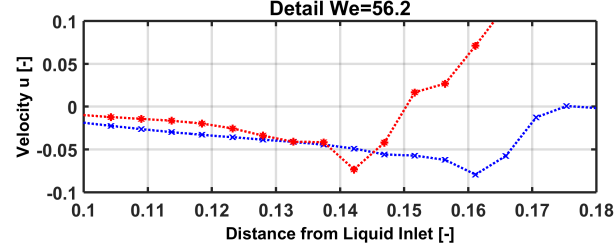

We $=52.0$
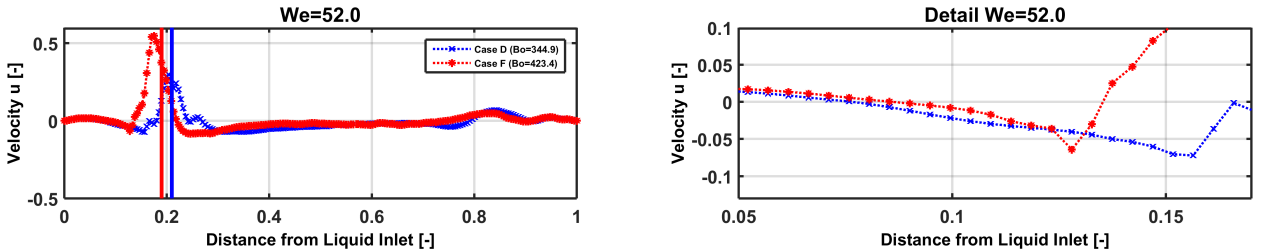

Figure 6: Plots of the transversal velocity component $v_{u}$. These velocity profiles are taken in the line parallel to the plate surface at a constant distance of $0.2 \mathrm{~mm}$ from it, and contained in the observation plane depicted in Figure 1 that is, at $5 \mathrm{~mm}$ from the lateral wall. The three subplots on the left hand side contain the values along the entire length of the plate. The range is restricted to the vicinity of the front wave in the three subplots on the right hand side so as to remark the difference between the data series. Each row of subplots contains data with the same liquid inlet velocity to assess the effect of gravity. The boundary between the liquid and the gas phase is represented by the vertical line of the same colour of its respective data series. 

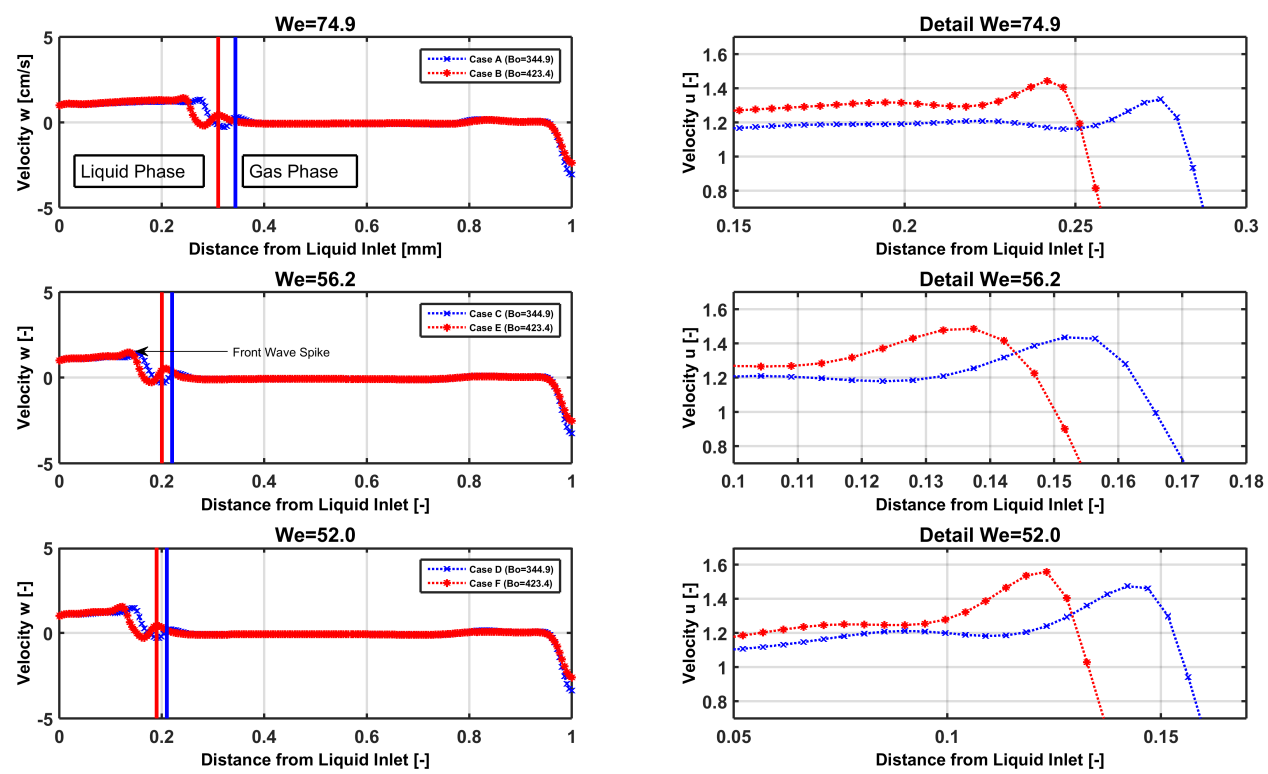

Figure 7: Plots of the transversal velocity component $v_{w}$. These velocity profiles are taken in the line parallel to the plate surface at a constant distance of $0.2 \mathrm{~mm}$ from it, and contained in the observation plane depicted in Figure 1 that is, at $5 \mathrm{~mm}$ from the lateral wall. The three subplots on the left hand side contain the values along the entire length of the plate. The range is restricted to the vicinity of the front wave in the three subplots on the right hand side so as to remark the difference between the data series. Each row of subplots contains data with the same liquid inlet velocity to assess the effect of gravity. The boundary between the liquid and the gas phase is represented by the vertical line of the same colour of its respective data series. 


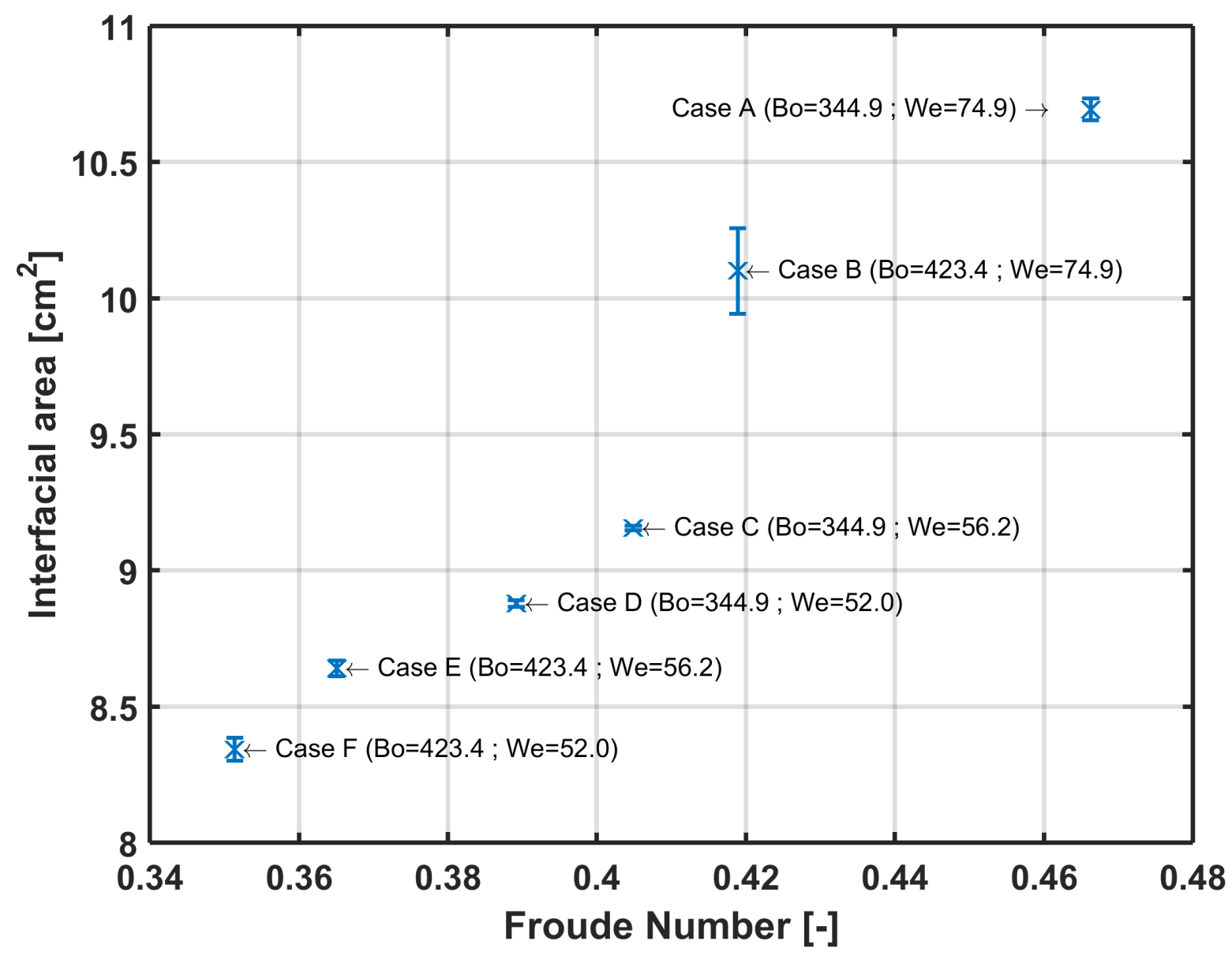

Figure 8: Interface area, obtained as the area of the isosurface with volume fraction $\gamma=0.5$, against the Froude number for the six cases tested. The data included correspond to the extrapolated values obtained using the Richardson extrapolation, and therefore grid discretisation error bars are included. 


\begin{tabular}{cccccc}
\hline Case & Velocity $[\mathrm{cm} / \mathrm{s}]$ & Inclination Hor. $\left.{ }^{\circ}\right]$ & $B o$ & $W e$ & $F r$ \\
\hline A & 30 & 45 & 344.9 & 74.9 & 0.4663 \\
B & 30 & 60 & 423.4 & 74.9 & 0.4189 \\
C & 26 & 45 & 344.9 & 56.2 & 0.4049 \\
D & 25 & 45 & 344.9 & 52.0 & 0.3892 \\
E & 26 & 60 & 423.4 & 56.2 & 0.3651 \\
F & 25 & 60 & 423.4 & 52.0 & 0.3514 \\
\hline
\end{tabular}

Table 1: Conditions of the $3 \times 2$ factorial experiment. Three levels are considered for the velocity and two for the inclination of the plate. The values of the Reynolds number $(R e)$, the Bond number $(B o)$, the Weber number $(W e)$, and the Froude number $(F r)$ are also shown, respectively. 


\begin{tabular}{cccccc}
\hline Grid-Level & Nodes x-dir & Nodes y-dir & Nodes z-dir & Total Nodes & $h[\mu \mathrm{m}]$ \\
\hline 3 & 35 & 67 & 147 & 344715 & 16.7 \\
2 & 42 & 78 & 177 & 579852 & 13.8 \\
1 & 50 & 93 & 212 & 985800 & 11.4 \\
\hline
\end{tabular}

Table 2: Number of nodes in each direction and the total number of nodes in three grid-levels. 


\begin{tabular}{cccccccc}
\hline Case & $f_{3}\left[\mathrm{~cm}^{2}\right]$ & $f_{2}\left[\mathrm{~cm}^{2}\right]$ & $f_{1}\left[\mathrm{~cm}^{2}\right]$ & $f_{\text {extr }}\left[\mathrm{cm}^{2}\right]$ & $\mathrm{GCI}_{21}[\%]$ & $\mathrm{GCI}_{32}[\%]$ & Asymptotic Range? \\
\hline A & 11.3746 & 10.7866 & 10.6935 & 10.6760 & 0.3708 & 2.3214 & Yes $\rightarrow 0.9914$ \\
B & 11.0568 & 10.4662 & 10.1011 & 9.5100 & 1.5392 & 2.4031 & Yes $\rightarrow 0.9651$ \\
C & 9.3327 & 9.1771 & 9.1558 & 9.1524 & 0.0991 & 0.7220 & Yes $\rightarrow 0.9977$ \\
D & 8.9723 & 8.9073 & 8.8777 & 8.8529 & 0.1420 & 0.3108 & Yes $\rightarrow 0.9967$ \\
E & 9.1431 & 8.7065 & 8.6397 & 8.6277 & 0.3291 & 2.1355 & Yes $\rightarrow 0.9923$ \\
F & 8.7848 & 8.4413 & 8.3422 & 8.3020 & 0.5059 & 1.7329 & Yes $\rightarrow 0.9883$ \\
\hline
\end{tabular}

Table 3: Results of the application of the Richardson extrapolation for the interface area $f$. 
Summary of important notation 


\begin{tabular}{|c|c|}
\hline \multicolumn{2}{|c|}{ Latin Symbols } \\
\hline$e$ & Relative Error [-] \\
\hline$f$ & Interface Area $\left[\mathrm{m}^{2}\right]$ \\
\hline$\vec{g}$ & Acceleration of Gravity $\left[\mathrm{m} \cdot \mathrm{s}^{-2}\right]$ \\
\hline$G C I$ & Grid Convergence Index [-] \\
\hline$h$ & Grid Spacing $[\mathrm{m}]$ \\
\hline$l$ & Characteristic Length $[\mathrm{m}]$ \\
\hline$\vec{n}$ & Normal Unit Vector to Interface \\
\hline$N$ & Total Number of Nodes in Grid [-] \\
\hline$p$ & Apparent Order of Convergence [-] \\
\hline$r$ & Grid Refinement Ratio [-] \\
\hline$S_{\sigma}$ & Momentum Source Term due to Surface Tension $\left[\mathrm{kg} \cdot \mathrm{m}^{-2} \cdot \mathrm{s}^{-2}\right]$ \\
\hline$t$ & Time $[\mathrm{s}]$ \\
\hline$u$ & Velocity $\left[\mathrm{m} \cdot \mathrm{s}^{-1}\right]$ \\
\hline \multicolumn{2}{|c|}{ Greek Symbols } \\
\hline$\alpha$ & Inclination of the plate over the horizontal plane $\left[^{\circ}\right]$ \\
\hline$\gamma$ & Volume Fraction [-] \\
\hline$\epsilon$ & Absolute Error \\
\hline$\theta$ & Contact Angle [-] \\
\hline$\kappa$ & Gas-Liquid Interface Curvature $\left[\mathrm{m}^{-1}\right]$ \\
\hline$\mu$ & Dynamic Viscosity $\left[\mathrm{kg} \cdot \mathrm{m}^{-1} \cdot \mathrm{s}^{-1}\right]$ \\
\hline$\rho$ & Density $\left[\mathrm{kg} \cdot \mathrm{m}^{-3}\right]$ \\
\hline$\sigma$ & Surface Tension Coefficient $\left[\mathrm{N} \cdot \mathrm{m}^{-1}\right]$ \\
\hline$\Phi$ & Generic Flow Variable \\
\hline \multicolumn{2}{|c|}{ Subscripts and Superscripts } \\
\hline $1,2,3$ & Refers to fine, medium and coarse mesh respectively \\
\hline eff & Effective. Used to correct gravity in Bond number \\
\hline extr & Extrapolated Value \\
\hline$g$ & Refers to Gas Phase \\
\hline$l$ & Refers to Liquid Phase \\
\hline$T$ & Superscript. Transposed matrix \\
\hline
\end{tabular}

